\title{
Democratic Republic of Timor-Leste: Statistical Appendix
}

This Statistical Appendix paper for the Democratic Republic of Timor-Leste was prepared by a staff team of the International Monetary Fund as background documentation for the periodic consultation with the member country. It is based on the information available at the time it was completed on June 29, 2004. The views expressed in this document are those of the staff team and do not necessarily reflect the views of the government of Democratic Republic of Timor-Leste or the Executive Board of the IMF.

The policy of publication of staff reports and other documents by the IMF allows for the deletion of market-sensitive information.

To assist the IMF in evaluating the publication policy, reader comments are invited and may be sent by e-mail to publicationpolicy@imf.org.

Copies of this report are available to the public from

International Monetary Fund • Publication Services

700 19th Street, N.W. • Washington, D.C. 20431

Telephone: (202) 6237430 • Telefax: (202) 6237201

E-mail: publications@imf.org • Internet: http://www.imf.org

Price: $\$ 15.00$ a copy

\section{International Monetary Fund} Washington, D.C. 



\title{
INTERNATIONAL MONETARY FUND \\ DEMOCRATIC REPUBLIC OF TIMOR-LESTE
}

\begin{abstract}
Statistical Appendix ${ }^{1}$
Prepared by Helene Poirson, Varapat Chensavasdijai, and Masahiro Nozaki

Approved by the Asia and Pacific Department
\end{abstract}

June 29, 2004

Contents

Page

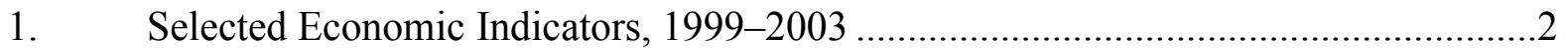

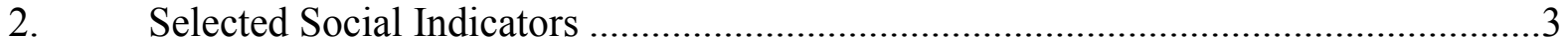

3. Gross Domestic Product by Sectoral Origin, 1998-2003 .......................................4

4. Gross Domestic Product by Expenditure, 1998-2003 ..........................................5

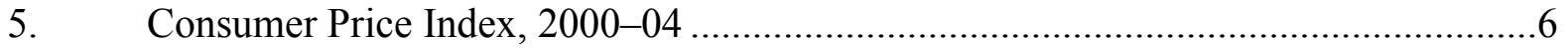

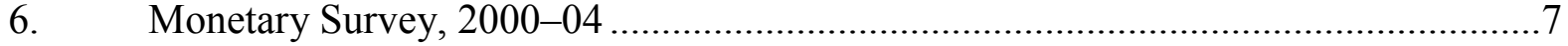

7. Balance sheet of the Banking and Payments Authority, 2000-04 ............................8

8. Consolidated Balance Sheet of Commercial Banks, 2000-04 ................................9

9. Central Government Budget Operations (CFET) FY2000/01-2004/05 ...................10

10. Central Government Domestic Revenues, FY2000/01-2004/05 …........................11

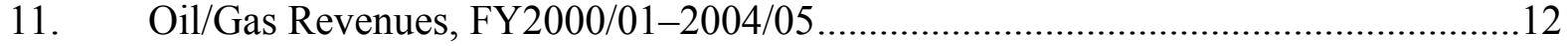

12. Central Government Expenditure Composition, FY2001/02-2004/05 .....................13

13. Public Sector Employment and Wages, FY2000/01-2004/05 .................................14

14. Autonomous Agencies Operations, FY2000/01-2004/05 .....................................15

15. Operations of the Power Authority, FY2000/01-2004/05 …................................16

16. Combined Sources of Fiscal Operations, FY2000/01-FY2003/04 ….....................17

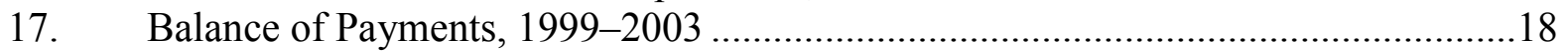

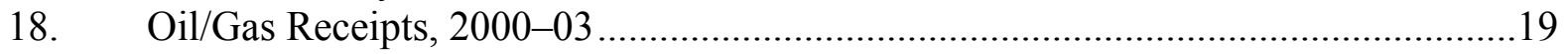

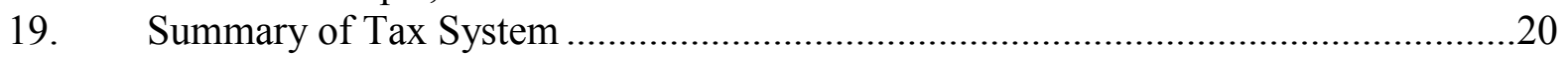

20. Summary of the Exchange and Payments System .............................................25

\footnotetext{
${ }^{1}$ Background information on key issues associated with the Timor-Leste economy, including the National Development Plan, the development of oil/gas resources, public finance, monetary developments, and the power sector, is provided in the Selected Issues and Statistical Appendix (IMF Country Report No. 03/228), which was issued in connection with the 2003 Article IV consultation.
} 
Table 1. Timor-Leste: Selected Economic Indicators, 1999-2003

\begin{tabular}{|c|c|c|c|c|c|}
\hline & 1999 & 2000 & 2001 & 2002 & 2003 \\
\hline & & & Est. & & \\
\hline \multicolumn{6}{|l|}{ Output and prices } \\
\hline GNP at current prices (in millions of U.S. dollars) & 270 & 329 & 400 & 397 & 372 \\
\hline GDP & 270 & 321 & 387 & 381 & 341 \\
\hline Oil/gas income & 0 & 8 & 13 & 17 & 31 \\
\hline Real GDP growth (percentage change) & -35 & 15 & 15 & 3 & -3 \\
\hline Inflation (percentage change at end-period) $1 /$ & 140 & 3 & 0 & 10 & 4 \\
\hline & \multicolumn{5}{|c|}{ (In percent of GDP) } \\
\hline \multicolumn{6}{|l|}{ Investment-saving balance } \\
\hline Gross investment $2 /$ & 21 & 33 & 31 & 29 & 27 \\
\hline Gross national savings & -13 & -53 & -47 & -43 & -32 \\
\hline External savings & 34 & 85 & 78 & 73 & 60 \\
\hline \multicolumn{6}{|l|}{ Government budget (CFET) 3/ } \\
\hline Revenues & $\ldots$ & 7.7 & 8.1 & 13.5 & 17.7 \\
\hline Domestic revenues & $\ldots$ & 4.0 & 5.3 & 5.3 & 8.2 \\
\hline Oil/gas revenues & $\ldots$ & 3.7 & 2.8 & 8.2 & 9.5 \\
\hline Expenditure & $\ldots$ & 14.5 & 13.7 & 19.6 & 22.1 \\
\hline Recurrent expenditure & $\ldots$ & 8.4 & 10.7 & 15.6 & 19.0 \\
\hline Capital expenditure & $\ldots$ & 6.1 & 3.0 & 4.0 & 3.1 \\
\hline Overall balance & $\ldots$ & -6.8 & -5.5 & -6.1 & -4.4 \\
\hline \multicolumn{6}{|l|}{ Combined sources fiscal operations $3 / 4 /$} \\
\hline Revenues & $\ldots$ & 8 & 9 & 15 & 19 \\
\hline Expenditure & $\ldots$ & 122 & 121 & 105 & 91 \\
\hline Recurrent expenditure & $\ldots$ & 96 & 97 & 82 & 72 \\
\hline Capital expenditure & $\ldots$ & 26 & 23 & 22 & 19 \\
\hline Overall balance & $\ldots$ & -114 & -111 & -90 & -72 \\
\hline \multicolumn{6}{|l|}{ Money and credit } \\
\hline Broad money (end-period) 5/ & 48 & 6 & 13 & 14 & 21 \\
\hline Net domestic assets (end-period) & 47 & -4 & -4 & -9 & -11 \\
\hline & \multicolumn{5}{|c|}{ (In millions of U.S. dollars) } \\
\hline \multicolumn{6}{|l|}{ External sector } \\
\hline Current account excl. official transfers & -92 & -279 & -309 & -292 & -230 \\
\hline Current account incl. official transfers & 6 & 48 & 54 & 44 & 43 \\
\hline Trade balance & -67 & -235 & -264 & -245 & -195 \\
\hline Merchandise exports 6/7/ & 52 & 5 & 4 & 6 & 7 \\
\hline Merchandise imports 6/ & -119 & -240 & -268 & -251 & -203 \\
\hline Overall balance & 0 & 16 & 8 & 20 & 18 \\
\hline & \multicolumn{5}{|c|}{ (In percent of GDP) } \\
\hline Current account excl. official transfers & -34 & -87 & -80 & -77 & -67 \\
\hline Current account incl. official transfers & 2 & 15 & 14 & 12 & 13 \\
\hline Trade balance & -25 & -73 & -68 & -64 & -57 \\
\hline Merchandise exports 6/7/ & 19 & 2 & 1 & 2 & 2 \\
\hline Merchandise imports 6/ & -44 & -75 & -69 & -66 & -59 \\
\hline Overall balance & 0 & 5 & 2 & 5 & 5 \\
\hline
\end{tabular}

Sources: Data provided by the Timor-Leste authorities; and Fund staff estimates.

1/ Rupiah-based CPI for Dili through 2000 and, thereafter, dollar-based CPI for Dili.

2/ Excludes investment relating to the oil/gas sector.

3/ On the basis of fiscal year (July-June); for example, 2000 relates to FY2000/01.

4/ Include fiscal and quasi-fiscal expenditure programs undertaken by bilateral donors and international financial institutions outside the central government budget.

5/ Figures after 1999 exclude currency holdings by the public, on which no data are available.

6/ Figures before 2000 include unrecorded border trade.

7/ Excludes oil/gas revenues, which are recorded under the income account (royalties) and transfers (tax revenues). 
Table 2. Timor-Leste: Selected Social Indicators 1/

\begin{tabular}{|c|c|c|c|}
\hline & $\begin{array}{l}\text { Timor- } \\
\text { Leste }\end{array}$ & $\begin{array}{l}\text { East Asia } \\
\text { and Pacific } \\
\text { island } \\
\text { countries }\end{array}$ & $\begin{array}{c}\text { Low } \\
\text { income } \\
\text { countries }\end{array}$ \\
\hline Per capita income (U.S. dollars, 2003) & 415 & 1,267 & 432 \\
\hline Area (in thousands of square kilometers) & 15 & $\ldots$ & $\ldots$ \\
\hline \multicolumn{4}{|l|}{ Demography } \\
\hline Total population (in thousands) & 825 & $1,838,371$ & $2,495,033$ \\
\hline Population growth (in percent) & $1.92 /$ & 1 & 2 \\
\hline \multicolumn{4}{|l|}{ Life expectancy and mortality } \\
\hline Life expectancy at birth (years) & 57 & 69 & 59 \\
\hline Male & 56 & 68 & 58 \\
\hline Female & 59 & 71 & 60 \\
\hline Infant mortality (per thousand live births) & 80 & 33 & 81 \\
\hline Male & 88 & $\ldots$ & $\ldots$ \\
\hline Female & 72 & $\ldots$ & $\ldots$ \\
\hline Under 5 mortality rate (per thousand live births) & 144 & 41 & 115 \\
\hline Male & 156 & $\ldots$ & $\ldots$ \\
\hline Female & 132 & $\cdots$ & .. \\
\hline \multicolumn{4}{|l|}{ Education } \\
\hline Illiteracy rate (in percent) $3 /$ & 57 & 13 & 37 \\
\hline Male & 57 & 7 & 28 \\
\hline Female & 57 & 18 & 47 \\
\hline Net primary school enrollment rate (in percent) & 76 & 93 & 74 \\
\hline \multicolumn{4}{|l|}{ Health } \\
\hline Number of hospitals (per thousand) & 0.5 & $\ldots$ & $\ldots$ \\
\hline Number of doctors (per thousand) & 0.2 & $\ldots$ & $\ldots$ \\
\hline Number of nurses (per thousand) & 1.4 & $\cdots$ & $\cdots$ \\
\hline \multicolumn{4}{|l|}{ Other indicators } \\
\hline The population below the poverty line $4 /$ & 41 & 29 & $\ldots$ \\
\hline Households with access to electricity 4/ & 36 & $\ldots$ & $\ldots$ \\
\hline Households with access to drinking water 4/ & 65 & 75 & 76 \\
\hline
\end{tabular}

Sources: UNDP Human Development Report 2003, World Bank, and Fund staff estimates.

1/ Relate to 2002, unless otherwise indicated.

2/ Average rate for the 1990s.

3/ Relates to the population of ages 15 and above.

4/ In percentage share of the total. 
Table 3. Timor-Leste: Gross Domestic Product by Sectoral Origin, 1998-2003 1/

\begin{tabular}{|c|c|c|c|c|c|c|}
\hline & 1998 & 1999 & 2000 & 2001 & 2002 & 2003 \\
\hline & \multicolumn{6}{|c|}{ Est. } \\
\hline & \multicolumn{6}{|c|}{ (At current market prices) } \\
\hline Agriculture, forestry, and fishery & 160.6 & 116.6 & 83.3 & 99.9 & 104.6 & 104.0 \\
\hline Mining and quarrying & 2.5 & 2.7 & 3.1 & 3.4 & 3.6 & 3.8 \\
\hline Manufacturing & 10.7 & 7.5 & 8.7 & 9.8 & 10.5 & 11.0 \\
\hline Electricity, gas, water & 3.0 & 2.1 & 2.6 & 3.1 & 3.2 & 3.2 \\
\hline Construction & 41.3 & 33.0 & 45.9 & 55.1 & 57.5 & 55.9 \\
\hline Trade, hotels, and restaurants & 28.4 & 15.1 & 25.1 & 32.7 & 33.4 & 29.9 \\
\hline Transport and communications & 47.0 & 14.6 & 23.2 & 29.2 & 30.1 & 27.5 \\
\hline Finance, rents, and business services & 15.1 & 10.8 & 21.1 & 26.7 & 26.5 & 23.8 \\
\hline Public admin. and defense 2/ & 78.3 & 66.2 & 106.3 & 125.0 & 108.7 & 79.9 \\
\hline Private services & 3.5 & 1.6 & 1.9 & 2.4 & 2.5 & 2.3 \\
\hline \multirow[t]{2}{*}{ Total } & 390.4 & 270.1 & 321.2 & 387.4 & 380.7 & 341.2 \\
\hline & \multicolumn{6}{|c|}{ (At constant 2000 market prices) } \\
\hline Agriculture, forestry, and fishery & 98.7 & 95.1 & 83.3 & 96.0 & 96.7 & 92.5 \\
\hline Mining and quarrying & 3.6 & 3.1 & 3.1 & 3.2 & 3.3 & 3.4 \\
\hline Manufacturing & 15.9 & 7.9 & 8.7 & 9.4 & 9.7 & 9.8 \\
\hline Electricity, gas, water & 4.7 & 2.3 & 2.6 & 3.0 & 3.0 & 2.9 \\
\hline Construction & 47.3 & 34.4 & 45.9 & 52.8 & 53.0 & 49.8 \\
\hline Trade, hotels, and restaurants & 40.5 & 17.4 & 25.1 & 31.2 & 30.7 & 26.7 \\
\hline Transport and communications & 37.4 & 15.9 & 23.2 & 27.9 & 27.7 & 24.6 \\
\hline Finance, rents, and business services & 38.3 & 16.9 & 21.1 & 25.4 & 24.4 & 21.2 \\
\hline Public admin. and defense 2/ & 141.8 & 83.5 & 106.3 & 116.9 & 128.2 & 135.9 \\
\hline Private services & 2.9 & 1.7 & 1.9 & 2.3 & 2.3 & 2.1 \\
\hline Total & 431.0 & 278.2 & 321.2 & 368.2 & 379.1 & 368.7 \\
\hline
\end{tabular}

Sources: Data provided by the Timor Leste authorities; and Fund staff estimates.

1/ Figures prior to 1999 are based on data provided by the Indonesian authorities. Figures for 2000 are partially based on estimates made under a World Bank-supported technical assistance project.

2/ Includes the value-added of services provided by UNTAET/UNMISET and donor-supported agencies acting on behalf of the Timor-Leste government. 
Table 4. Timor-Leste: Gross Domestic Product by Expenditure, 1998-2003 1/

\begin{tabular}{|c|c|c|c|c|c|c|}
\hline & 1998 & 1999 & 2000 & 2001 & 2002 & 2003 \\
\hline & \multicolumn{6}{|c|}{ Est. } \\
\hline & \multicolumn{6}{|c|}{ (In millions of U.S. dollars) } \\
\hline GDP at current prices & 390.4 & 270.1 & 321.2 & 387.4 & 380.7 & 341.2 \\
\hline Consumption & 375.1 & 305.3 & 498.1 & 580.4 & 562.6 & 483.0 \\
\hline Private & 316.5 & 264.8 & 308.1 & 361.1 & 352.1 & 310.9 \\
\hline Public 2/3/ & 58.6 & 40.5 & 190.0 & 219.4 & 210.5 & 172.1 \\
\hline Gross investment 4/ & 136.6 & 56.7 & 105.7 & 120.7 & 111.3 & 93.6 \\
\hline Private & 39.0 & 13.5 & 24.3 & 30.2 & 27.6 & 23.0 \\
\hline Public 2/ & 97.6 & 43.2 & 81.5 & 90.5 & 83.7 & 70.6 \\
\hline Net exports of goods/non-factor services & -121.3 & -91.9 & -282.6 & -313.8 & -293.2 & -235.4 \\
\hline GNP at current prices & 390.4 & 270.1 & 329.3 & 399.9 & 397.3 & 372.4 \\
\hline GDP & 390.4 & 270.1 & 321.2 & 387.4 & 380.7 & 341.2 \\
\hline Oil income & 0.0 & 0.0 & 8.1 & 12.5 & 16.5 & 31.2 \\
\hline Gross national savings & 15.4 & -35.2 & -168.8 & -180.5 & -165.3 & -110.6 \\
\hline Private & 23.2 & -32.5 & 4.5 & 9.0 & 8.8 & 9.6 \\
\hline Public 2/ & -7.8 & -2.7 & -173.3 & -189.5 & -174.1 & -120.1 \\
\hline \multirow[t]{2}{*}{ External savings } & 121.3 & 91.9 & 274.5 & 301.3 & 276.6 & 204.2 \\
\hline & \multicolumn{6}{|c|}{ (In percent of GDP) } \\
\hline GDP at current prices & 100.0 & 100.0 & 100.0 & 100.0 & 100.0 & 100.0 \\
\hline Consumption & 96.1 & 113.0 & 155.1 & 149.8 & 147.8 & 141.6 \\
\hline Private & 81.1 & 98.0 & 95.9 & 93.2 & 92.5 & 91.1 \\
\hline Public 2/3/ & 15.0 & 15.0 & 59.2 & 56.6 & 55.3 & 50.5 \\
\hline Gross investment 4/ & 35.0 & 21.0 & 32.9 & 31.2 & 29.2 & 27.4 \\
\hline Private & 10.0 & 5.0 & 7.6 & 7.8 & 7.2 & 6.7 \\
\hline Public 2/ & 25.0 & 16.0 & 25.4 & 23.4 & 22.0 & 20.7 \\
\hline Net exports of goods/non-factor services & -31.1 & -34.0 & -88.0 & -81.0 & -77.0 & -69.0 \\
\hline GNP at current prices & 100.0 & 100.0 & 102.5 & 103.2 & 104.3 & 109.1 \\
\hline GDP & 100.0 & 100.0 & 100.0 & 100.0 & 100.0 & 100.0 \\
\hline Oil income & 0.0 & 0.0 & 2.5 & 3.2 & 4.3 & 9.1 \\
\hline Gross national savings & 3.9 & -13.0 & -52.6 & -46.6 & -43.4 & -32.4 \\
\hline Private & 5.9 & -12.0 & 1.4 & 2.3 & 2.3 & 2.8 \\
\hline Public 2/ & -2.0 & -1.0 & -53.9 & -48.9 & -45.7 & -35.2 \\
\hline External savings & 31.1 & 34.0 & 85.5 & 77.8 & 72.7 & 59.9 \\
\hline
\end{tabular}

Sources: Data provided by the Timor-Leste authorities; and Fund staff estimates.

1/ Figures prior to 1999 are based on data provided by the Indonesian authorities. Figures for 2000 are based partially on estimates made under a World Bank-supported technical assistance project.

2 / Includes fiscal and quasi-fiscal activities undertaken by multinational institutions and bilateral donors.

3/ Does not necessarily correspond to recurrent expenditures shown in Table 16 due to differences in the coverage of data (particularly those relating to technical assistance).

4/ Excludes investment relating to the oil/gas sector. 


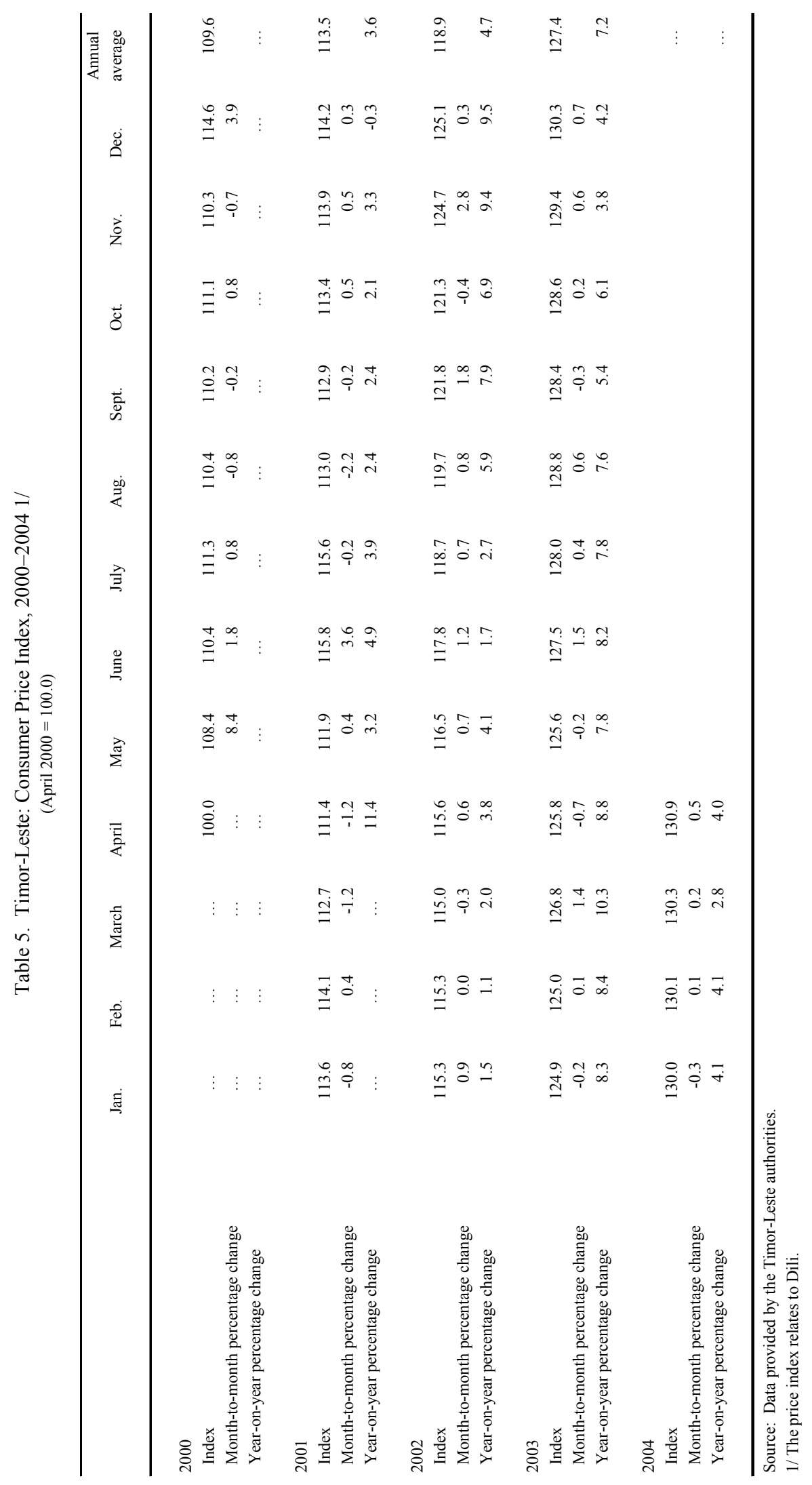




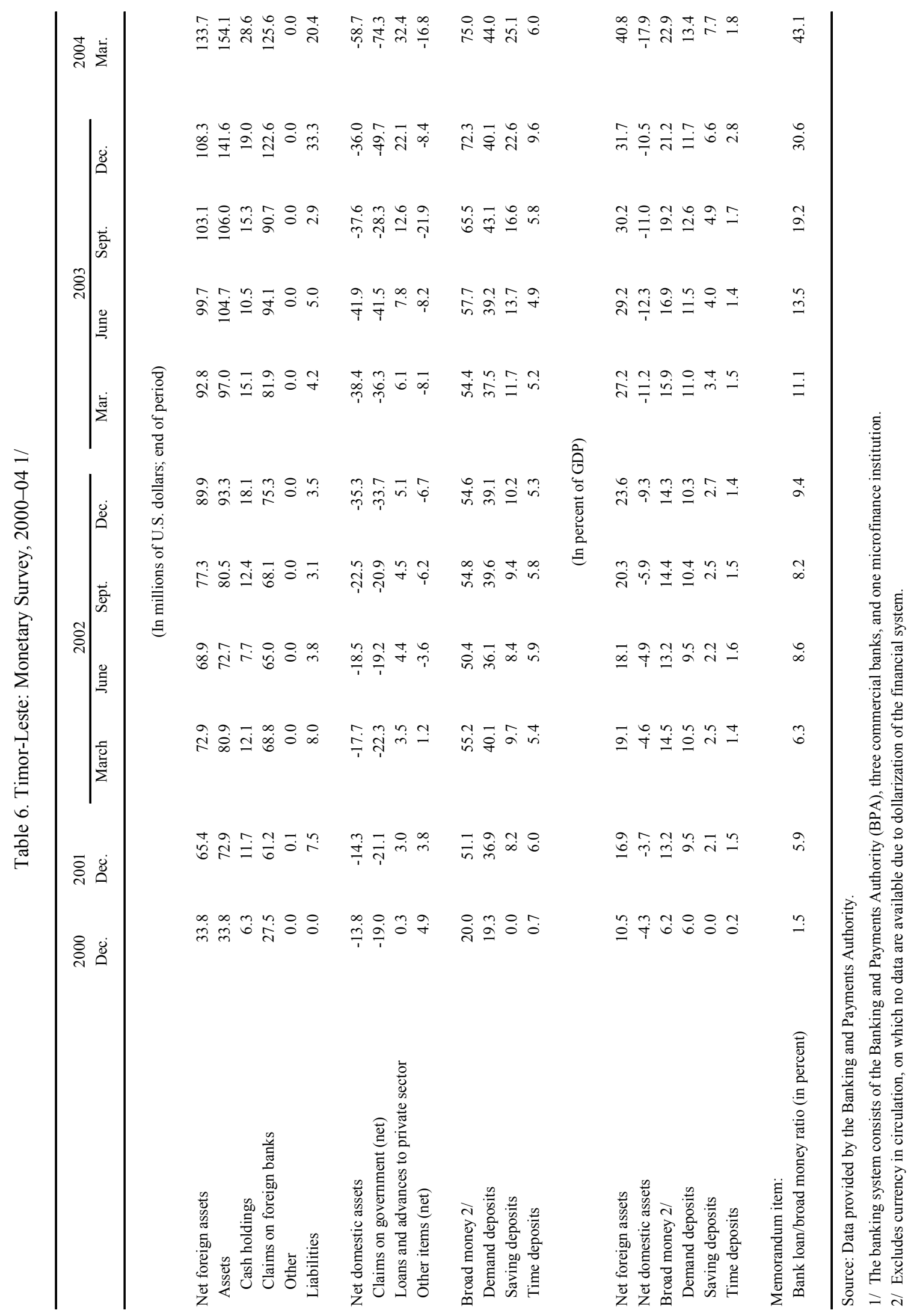




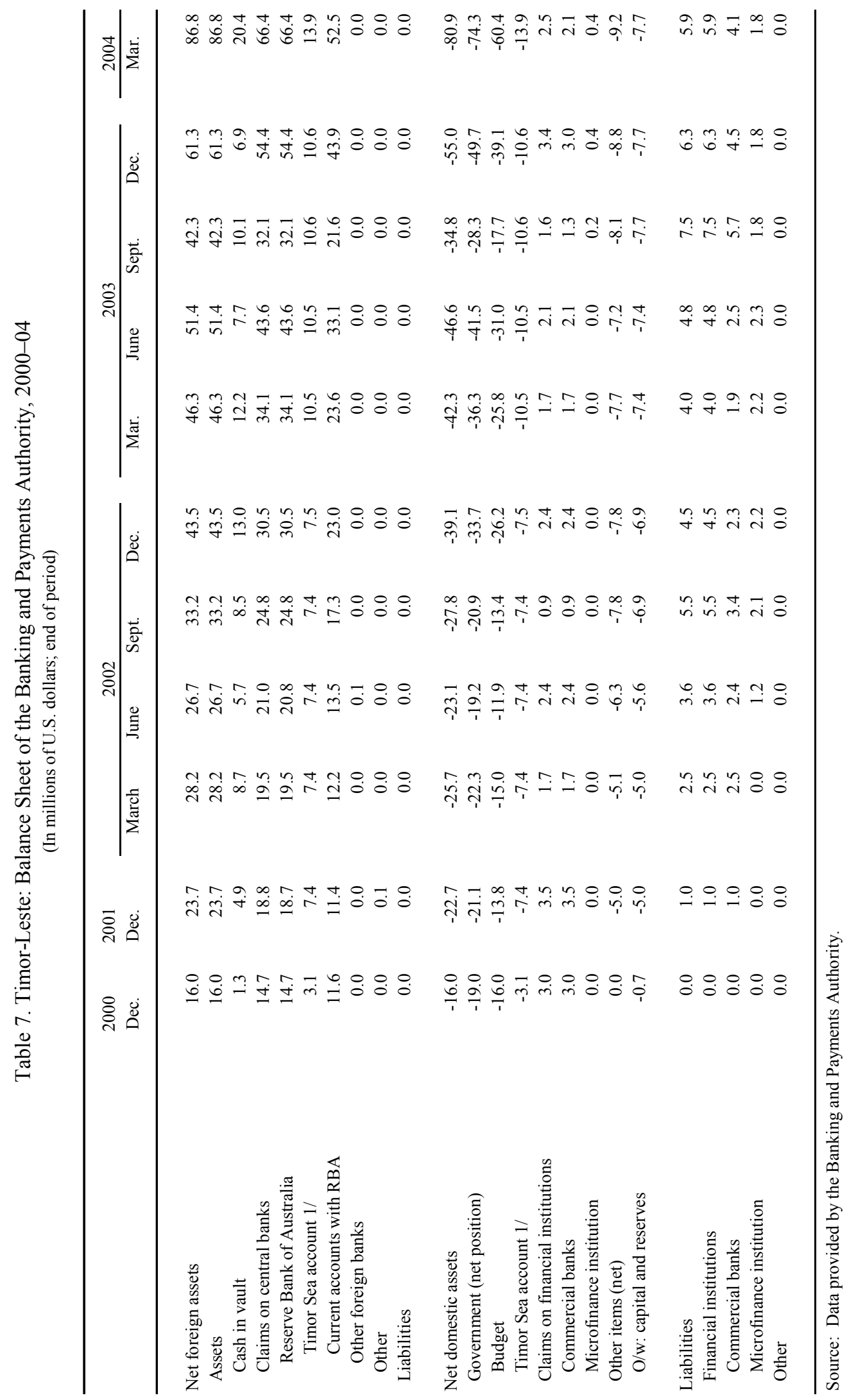




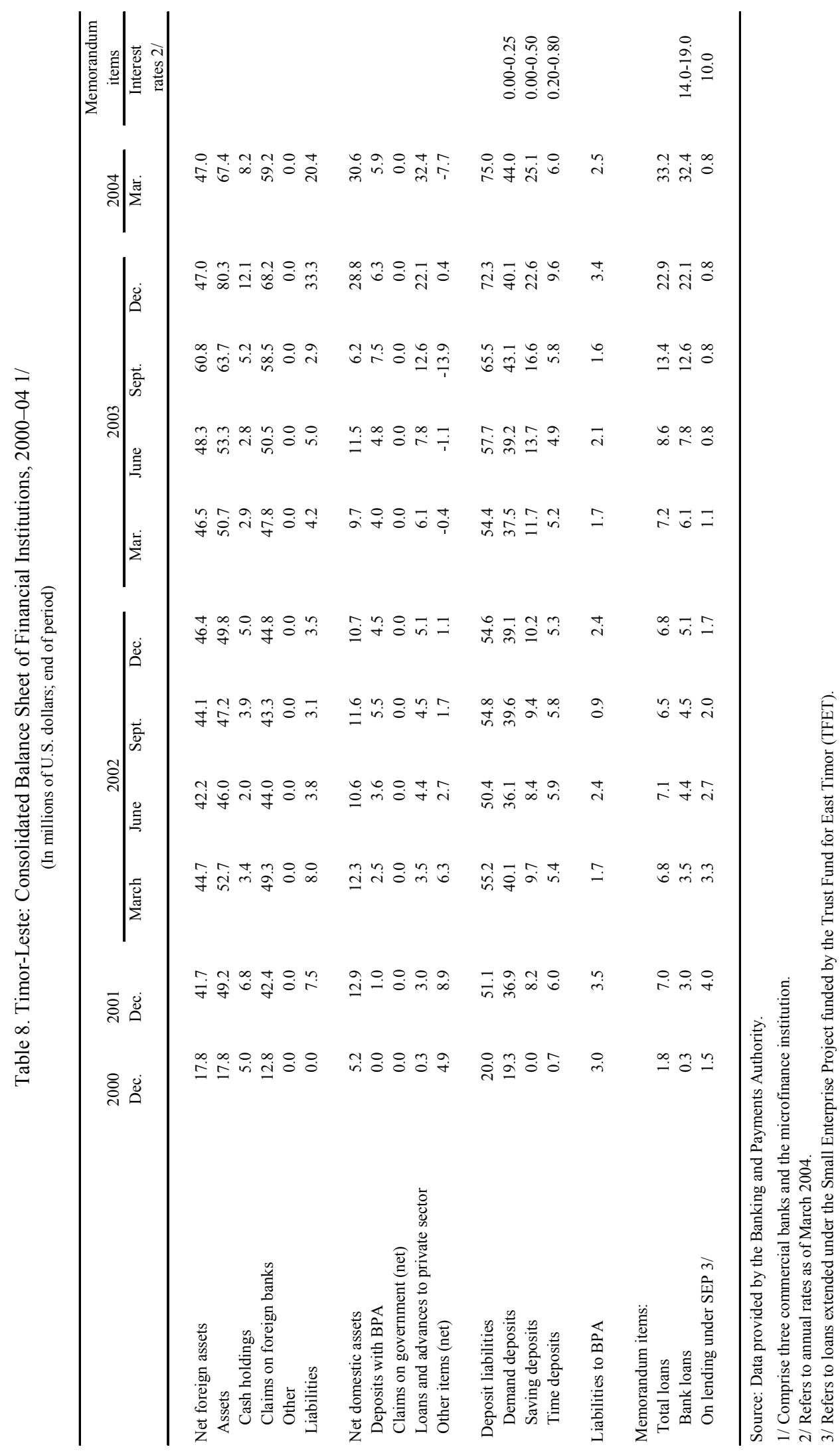


Table 9. Timor-Leste: Central Government Budget Operations (CFET), FY2000/01-FY2004/05 1/

\begin{tabular}{|c|c|c|c|c|c|c|c|}
\hline & \multirow[t]{2}{*}{ FY2000/01 } & \multirow[t]{2}{*}{ FY2001/02 } & \multirow[t]{2}{*}{ FY2002/03 } & \multicolumn{3}{|c|}{ FY2003/04 } & \multirow{2}{*}{$\frac{\text { FY2004/05 }}{\text { Budget }}$} \\
\hline & & & & Budget & Revised 2/ & Est. 3/ & \\
\hline & \multicolumn{6}{|c|}{ (In millions of U.S. dollars) } & \\
\hline Revenue & 27.1 & 31.3 & 48.8 & 59.8 & 45.1 & 59.3 & 67.2 \\
\hline Domestic revenues & 14.1 & 20.5 & 19.3 & 17.6 & 20.3 & 27.5 & 23.0 \\
\hline Direct taxes & 0.6 & 5.4 & 5.3 & 4.7 & 5.4 & 6.1 & 5.4 \\
\hline Indirect taxes & 11.6 & 12.7 & 11.7 & 10.2 & 11.8 & 16.9 & 13.6 \\
\hline Nontax revenues and other & 1.9 & 2.4 & 2.3 & 2.7 & 3.1 & 4.5 & 4 \\
\hline Oil/gas revenues & 13.1 & 10.8 & 29.5 & 42.2 & 24.8 & 31.8 & 44.2 \\
\hline Tax revenues & 9.9 & 6.5 & 26.4 & 29.3 & 17.1 & 27.2 & 18.3 \\
\hline Royalties and interest & 3.1 & 4.3 & 3.1 & 12.9 & 7.7 & 4.6 & 25.8 \\
\hline Royalties & 3.0 & 4.2 & 3.0 & 12.3 & 7.6 & 4.5 & 25.5 \\
\hline Interest & 0.1 & 0.1 & 0.1 & 0.6 & 0.1 & 0.1 & 0.3 \\
\hline Expenditure & 51.3 & 52.6 & 70.8 & 79.1 & 74.6 & 74.1 & 75.1 \\
\hline Recurrent expenditure & 29.6 & 41.1 & 56.2 & 66.8 & 64.2 & 63.7 & 64.9 \\
\hline Wages and salaries & 13.9 & 18.8 & 21.9 & 27.0 & 26.1 & 25.6 & 28.2 \\
\hline Goods and services & 15.7 & 22.2 & 34.3 & 39.8 & 38.1 & 38.1 & 36.7 \\
\hline Capital expenditure & 21.7 & 11.5 & 14.5 & 12.3 & 10.4 & 10.4 & 10.2 \\
\hline Overall balance & -24.2 & -21.3 & -22.0 & -19.3 & -29.5 & -14.8 & -7.9 \\
\hline Financing & 24.2 & 21.3 & 22.0 & 19.3 & 29.6 & 14.8 & 7.9 \\
\hline Grants 4/ & 31.6 & 22.7 & 32.5 & 28.0 & 33.8 & 35.6 & 30.8 \\
\hline Changes in CFET cash balances (increase -) & -4.2 & 2.7 & -8.6 & 4.2 & 3.5 & -16.2 & 3.0 \\
\hline Oil/gas revenue savings (increase -) 5/ & -3.1 & -4.3 & -3.1 & -12.9 & -7.7 & -4.6 & -25.8 \\
\hline \multirow[t]{2}{*}{ Other } & -0.1 & 0.2 & 1.1 & 0.0 & 0.0 & 0.0 & 0.0 \\
\hline & \multicolumn{7}{|c|}{ (In percent of GDP) } \\
\hline Revenue & 7.7 & 8.1 & 13.5 & 18.0 & 13.5 & 17.7 & 20.4 \\
\hline Domestic revenues & 4.0 & 5.3 & 5.3 & 5.3 & 6.1 & 8.2 & 7.0 \\
\hline Direct taxes & 0.2 & 1.4 & 1.5 & 1.4 & 1.6 & 1.8 & 1.6 \\
\hline Indirect taxes & 3.3 & 3.3 & 3.2 & 3.1 & 3.5 & 5.0 & 4.1 \\
\hline Nontax revenues and other & 0.5 & 0.6 & 0.6 & 0.8 & 0.9 & 1.4 & 1.2 \\
\hline Oil/gas revenues & 3.7 & 2.8 & 8.2 & 12.7 & 7.4 & 9.5 & 13.4 \\
\hline $\mathrm{O} / \mathrm{w}$ : Tax revenues & 2.8 & 1.7 & 7.3 & 8.8 & 5.1 & 8.1 & 5.6 \\
\hline Expenditure & 14.5 & 13.7 & 19.6 & 23.9 & 22.3 & 22.2 & 22.8 \\
\hline Recurrent expenditure & 8.4 & 10.7 & 15.6 & 20.1 & 19.2 & 19.0 & 19.7 \\
\hline Wages and salaries & 3.9 & 4.9 & 6.1 & 8.1 & 7.8 & 7.6 & 8.5 \\
\hline Goods and services & 4.4 & 5.8 & 9.5 & 12.0 & 11.4 & 11.4 & 11.1 \\
\hline Capital expenditure & 6.1 & 3.0 & 4.0 & 3.7 & 3.1 & 3.1 & 3.1 \\
\hline Overall balance & -6.8 & -5.5 & -6.1 & -5.8 & -8.8 & -4.4 & -2.4 \\
\hline \multirow[t]{2}{*}{ Financing } & 6.8 & 5.5 & 6.1 & 5.8 & 8.8 & 4.4 & 2.4 \\
\hline & \multicolumn{7}{|c|}{ (In millions of U.S. dollars unless otherwise indicated) } \\
\hline \multicolumn{8}{|l|}{ Memorandum items: } \\
\hline Cumulative oil/gas savings (end-period) & 3.1 & 7.4 & 10.5 & 22.4 & 18.3 & 15.1 & 41.0 \\
\hline (In percent of recurrent expenditure) & 10.6 & 18.1 & 18.7 & 33.6 & 28.4 & 23.8 & 63.1 \\
\hline CFET cash balances (end-period) 6/ & 8.9 & 6.3 & 14.8 & 6.0 & 11.3 & 31.0 & 28.0 \\
\hline Non-oil overall fiscal balance & -37.3 & -32.0 & -51.4 & -61.5 & -54.3 & -46.6 & -52.1 \\
\hline (In percent of GDP) & -10.5 & -8.3 & -14.3 & -18.6 & -16.2 & -13.9 & -15.8 \\
\hline
\end{tabular}

Sources: Data provided by the Timor-Leste authorities; and Fund staff estimates.

1/ Fiscal year: July-June.

2/ Revised estimates made at the mid-year budget review (November 2003).

3/ Staff estimates based on the actual outcome through March 2004.

4/ In the official presentation, grants are shown "below the line" to highlight the amount of donor assistance required to close financing gaps.

5/ Under the current oil/gas saving policy, royalties and interest income are automatically saved and only tax revenues are available for budget

financing.

6/ The budge estimate for FY2003/04 was derived on the basis of CFET cash balances ( $\$ 10.2$ million) projected during the 2002 mid-year

budget review. 
Table 10. Timor-Leste: Central Government Domestic Revenues, FY2000/01-FY2004/05 1/

\begin{tabular}{|c|c|c|c|c|c|c|c|}
\hline & \multirow[t]{2}{*}{ FY2000/01 } & \multirow[t]{2}{*}{ FY2001/02 } & \multirow[t]{2}{*}{ FY2002/03 } & \multicolumn{3}{|c|}{ FY2003/04 } & \multirow{2}{*}{$\frac{\text { FY2004/05 }}{\text { Budget }}$} \\
\hline & & & & Budget & Revised 2/ & Est. 3/ & \\
\hline & \multicolumn{7}{|c|}{ (In millions of U.S. dollars) } \\
\hline Total domestic revenue & 14.1 & 20.5 & 19.3 & 17.6 & 20.3 & 27.5 & 23.0 \\
\hline Tax revenue & 12.2 & 18.1 & 17.0 & 14.9 & 17.2 & 23.0 & 19.0 \\
\hline Direct tax & 0.6 & 5.4 & 5.3 & 4.7 & 5.4 & 6.1 & 5.4 \\
\hline Wage tax & 0.5 & 2.5 & 2.7 & 2.6 & 3.0 & 2.7 & 2.7 \\
\hline Government & 0.5 & 0.5 & 0.5 & 0.6 & 0.7 & 0.5 & 0.5 \\
\hline Other & 0.0 & 1.9 & 2.2 & 2.0 & 2.3 & 2.1 & 2.2 \\
\hline Corporate income tax & 0.2 & 1.7 & 1.7 & 1.3 & 1.5 & 1.8 & 1.2 \\
\hline Special withholding tax & 0.0 & 1.3 & 0.9 & 0.8 & 0.9 & 1.7 & 1.5 \\
\hline Indirect tax & 11.6 & 12.7 & 11.7 & 10.2 & 11.8 & 16.9 & 13.6 \\
\hline Tax on goods and services & 8.8 & 9.6 & 8.7 & 7.5 & 8.7 & 13.1 & 10.4 \\
\hline Sales tax 4/ & 3.0 & 3.3 & 3.4 & 3.0 & 3.4 & 4.1 & 3.3 \\
\hline Excise duty 4/ & 4.1 & 4.3 & 3.4 & 3.0 & 3.5 & 6.6 & 5.0 \\
\hline Service tax $5 /$ & 1.7 & 2.0 & 1.9 & 1.5 & 1.7 & 2.5 & 2.1 \\
\hline Tax on international trade & 2.8 & 3.1 & 3.0 & 2.7 & 3.1 & 3.8 & 3.2 \\
\hline Import duty & 2.6 & 3.1 & 3.0 & 2.7 & 3.1 & 3.8 & 3.2 \\
\hline Export duty 6/ & 0.2 & 0.0 & 0.0 & 0.0 & 0.0 & 0.0 & 0.0 \\
\hline Non-tax revenue & 1.9 & 2.4 & 2.3 & 2.7 & 3.1 & 4.5 & 4.0 \\
\hline Fees and charges & 1.2 & 1.8 & 2.1 & $\ldots$ & $\ldots$ & 4.1 & 3.7 \\
\hline $\mathrm{O} / \mathrm{w}$ : Property rental & 1.1 & 1.1 & 1.1 & $\ldots$ & $\ldots$ & 1.2 & 1.2 \\
\hline \multirow[t]{2}{*}{ Interest receipts and other } & 0.6 & 0.6 & 0.2 & $\ldots$ & $\ldots$ & 0.4 & 0.3 \\
\hline & \multicolumn{7}{|c|}{ (In percent of GDP) } \\
\hline Total domestic revenue & 4.0 & 5.3 & 5.3 & 5.3 & 6.1 & 8.2 & 7.0 \\
\hline Tax revenue & 3.4 & 4.7 & 4.7 & 4.5 & 5.1 & 6.9 & 5.8 \\
\hline Direct tax & 0.2 & 1.4 & 1.5 & 1.4 & 1.6 & 1.8 & 1.6 \\
\hline Wage tax & 0.1 & 0.6 & 0.7 & 0.8 & 0.9 & 0.8 & 0.8 \\
\hline Corporate income tax & 0.0 & 0.4 & 0.5 & 0.4 & 0.5 & 0.5 & 0.4 \\
\hline Special withholding tax & 0.0 & 0.3 & 0.2 & 0.2 & 0.3 & 0.5 & 0.5 \\
\hline Indirect tax & 3.3 & 3.3 & 3.2 & 3.1 & 3.5 & 5.0 & 4.1 \\
\hline Tax on goods and services & 2.5 & 2.5 & 2.4 & 2.3 & 2.6 & 3.9 & 3.2 \\
\hline Sales tax 4/ & 0.8 & 0.8 & 0.9 & 0.9 & 1.0 & 1.2 & 1.0 \\
\hline Excise duty 4/ & 1.2 & 1.1 & 1.0 & 0.9 & 1.1 & 2.0 & 1.5 \\
\hline Service tax 5/ & 0.5 & 0.5 & 0.5 & 0.5 & 0.5 & 0.7 & 0.6 \\
\hline Tax on international trade & 0.8 & 0.8 & 0.8 & 0.8 & 0.9 & 1.1 & 1.0 \\
\hline Non-tax revenue & 0.5 & 0.6 & 0.6 & 0.8 & 0.9 & 1.4 & 1.2 \\
\hline \multicolumn{8}{|l|}{ Memorandum item: } \\
\hline Taxes collected at the border & 9.9 & 10.7 & 9.8 & 8.7 & 10.0 & 14.4 & 11.5 \\
\hline (In percent of GDP) & 2.8 & 2.8 & 2.7 & 2.6 & 3.0 & 4.3 & 3.5 \\
\hline
\end{tabular}

Sources: Data provided by the Timor-Leste authorities and Fund staff calculations.

1/ Fiscal year: July-June.

2/ Revised estimates made at the mid-year budget review (November 2003).

3/ Staff estimates based on the actual outcome through March 2004.

4 / Currently collected only at the border.

5/ Turnover tax on hotel, restaurant, and transportation rental services.

6/ Abolished in July 2001. 
Table 11. Timor-Leste: Oil/gas Revenues, FY2000/01-FY2004/05 1/

\begin{tabular}{|c|c|c|c|c|c|c|c|}
\hline & \multirow[t]{2}{*}{ FY2000/01 } & \multirow[t]{2}{*}{ FY2001/02 } & \multirow[t]{2}{*}{ FY2002/03 } & \multicolumn{3}{|c|}{ FY2003/04 } & \multirow{2}{*}{$\frac{\text { FY2004/05 }}{\text { Budget }}$} \\
\hline & & & & Budget & Revised 2/ & Est. 3/ & \\
\hline & \multicolumn{7}{|c|}{ (In millions of U.S. dollars) } \\
\hline Total oil/gas revenues & 13.1 & 10.8 & 29.5 & 42.2 & 24.7 & 31.8 & 44.2 \\
\hline Tax revenues & 9.9 & 6.5 & 26.4 & 29.3 & 17.1 & 27.2 & 18.3 \\
\hline Income tax & 6.2 & 4.1 & 7.4 & 3.2 & 3.3 & 8.8 & 10.7 \\
\hline VAT & 3.1 & 1.7 & 10.9 & 20.7 & 15.8 & 12.3 & 8.2 \\
\hline Withholding tax & 0.7 & 0.7 & 6.7 & 3.8 & 3.4 & 4.1 & 1.1 \\
\hline Wage tax 4/ & 0.0 & 0.0 & 1.4 & 1.6 & 1.5 & 8.4 & 0.9 \\
\hline Tax refunding $5 /$ & 0.0 & 0.0 & 0.0 & 0.0 & -7.0 & -6.5 & -2.5 \\
\hline Royalties and interest & 3.1 & 4.3 & 3.1 & 12.9 & 7.7 & 4.6 & 25.8 \\
\hline Royalties & 3.0 & 4.2 & 3.0 & 12.3 & 7.6 & 4.5 & 25.5 \\
\hline \multirow[t]{2}{*}{ Interest income } & 0.1 & 0.1 & 0.1 & 0.6 & 0.1 & 0.1 & 0.3 \\
\hline & \multicolumn{7}{|c|}{ (In percent of GDP) } \\
\hline Total oil/gas revenues & 3.7 & 2.8 & 8.2 & 12.7 & 7.4 & 9.5 & 13.4 \\
\hline Tax revenues & 2.8 & 1.7 & 7.3 & 8.8 & 5.1 & 8.1 & 5.6 \\
\hline Income tax & 1.7 & 1.1 & 2.0 & 1.0 & 1.0 & 2.6 & 3.2 \\
\hline VAT & 0.9 & 0.4 & 3.0 & 6.2 & 4.7 & 3.7 & 2.5 \\
\hline Withholding tax & 0.2 & 0.2 & 1.9 & 1.1 & 1.0 & 1.2 & 0.3 \\
\hline Wage tax 4/ & 0.0 & 0.0 & 0.4 & 0.5 & 0.5 & 2.5 & 0.3 \\
\hline Tax refunding 5 / & 0.0 & 0.0 & 0.0 & 0.0 & -2.1 & -1.9 & -0.8 \\
\hline Royalties and interest & 0.9 & 1.1 & 0.9 & 3.9 & 2.3 & 1.4 & 7.8 \\
\hline Royalties & 0.9 & 1.1 & 0.8 & 3.7 & 2.3 & 1.3 & 7.7 \\
\hline Interest income & 0.0 & 0.0 & 0.0 & 0.2 & 0.0 & 0.0 & 0.1 \\
\hline \multicolumn{8}{|l|}{ Memorandum items: } \\
\hline Cumulative oil/gas savings (end-period) & 3.1 & 7.4 & 10.5 & 22.4 & 18.2 & 15.1 & 41.0 \\
\hline (In percent of GDP) & 0.9 & 1.9 & 2.9 & 6.8 & 5.4 & 4.5 & 12.4 \\
\hline
\end{tabular}

Sources: Data provided by the Timor-Leste authorities; and Fund staff estimates and calculations.

1/ Fiscal year: July -June.

2/ Revised estimates made at the mid-year budget review (November 2003).

3/ Staff estimates based on the actual outcome through March 2004.

4/ Wages paid by oil/gas companies operating in the Timor-Sea became taxable upon signing of the Timor Sea Treaty in May 2002.

5 / Due to the retroactive application of changes in the tax arrangement following the finalization of the Bayu-Undan project and other

ad-hoc factors. 
Table 12. Timor-Leste: Central Government Expenditure Composition, FY2001/02-FY2004/05 1/

\begin{tabular}{|c|c|c|c|c|c|c|}
\hline & \multirow[t]{2}{*}{ FY2001/02 } & \multirow[t]{2}{*}{ FY2002/03 } & \multicolumn{3}{|c|}{ FY2003/04 } & \multirow{2}{*}{$\frac{\text { FY2004/05 }}{\text { Budget }}$} \\
\hline & & & Budget & Revised 2/ & Est. 3/ & \\
\hline & \multicolumn{6}{|c|}{ (In millions of U.S. dollars) } \\
\hline Total expenditure & 52.6 & 70.8 & 79.1 & 74.6 & 74.1 & 75.1 \\
\hline Wages and salaries & 18.8 & 21.9 & 27.0 & 26.1 & 25.6 & 28.2 \\
\hline Salaries & 18.8 & 21.9 & 26.8 & 25.9 & 25.4 & 28.1 \\
\hline Overtime/allowances & 0.0 & 0.0 & 0.2 & 0.2 & 0.2 & 0.1 \\
\hline Goods and services & 22.2 & 34.3 & 39.8 & 38.1 & 38.1 & 36.7 \\
\hline Travel & 1.0 & 1.7 & 1.6 & 1.8 & 1.8 & 2.1 \\
\hline Vehicle & 1.6 & 4.6 & 4.3 & 4.2 & 4.2 & 4.1 \\
\hline Utilities & 0.4 & 1.5 & 2.3 & 2.3 & 2.3 & 2.5 \\
\hline Fuel for generators & 0.0 & 6.2 & 5.1 & 4.8 & 4.8 & 4.5 \\
\hline Materials and supplies & 6.5 & 8.4 & 8.7 & 8.2 & 8.2 & 7.5 \\
\hline Other operational expenses & 10.4 & 3.6 & 6.4 & 6.4 & 6.4 & 6.1 \\
\hline Training/workshops & 0.3 & 0.7 & 1.1 & 1.0 & 1.0 & 1.0 \\
\hline Services & 2.1 & 7.6 & 10.3 & 9.3 & 9.3 & 9.1 \\
\hline Capital expenditure & 11.5 & 14.5 & 12.3 & 10.4 & 10.4 & 10.2 \\
\hline Infrastructure & 1.7 & 5.1 & 7.4 & 7.0 & 7.0 & 8.4 \\
\hline Purchase of equipment & 6.0 & 4.7 & 3.3 & 2.1 & 2.1 & 1.3 \\
\hline \multirow[t]{2}{*}{ Other } & 3.8 & 4.7 & 1.6 & 1.3 & 1.3 & 0.6 \\
\hline & \multicolumn{6}{|c|}{ (In percent of GDP) } \\
\hline Total expenditure & 13.7 & 19.6 & 23.9 & 22.3 & 22.2 & 22.8 \\
\hline Wages and salaries & 4.9 & 6.1 & 8.1 & 7.8 & 7.6 & 8.5 \\
\hline Goods and services & 5.8 & 9.5 & 12.0 & 11.4 & 11.4 & 11.1 \\
\hline Capital expenditure & 3.0 & 4.0 & 3.7 & 3.1 & 3.1 & 3.1 \\
\hline
\end{tabular}

Sources: Data provided by the Timor-Leste Authorities.

1/ Fiscal year: July June.

2/ Revised estimates made at the mid-year budget review (November 2003).

3/ Staff estimates based on the actual outcome through March 2004. 


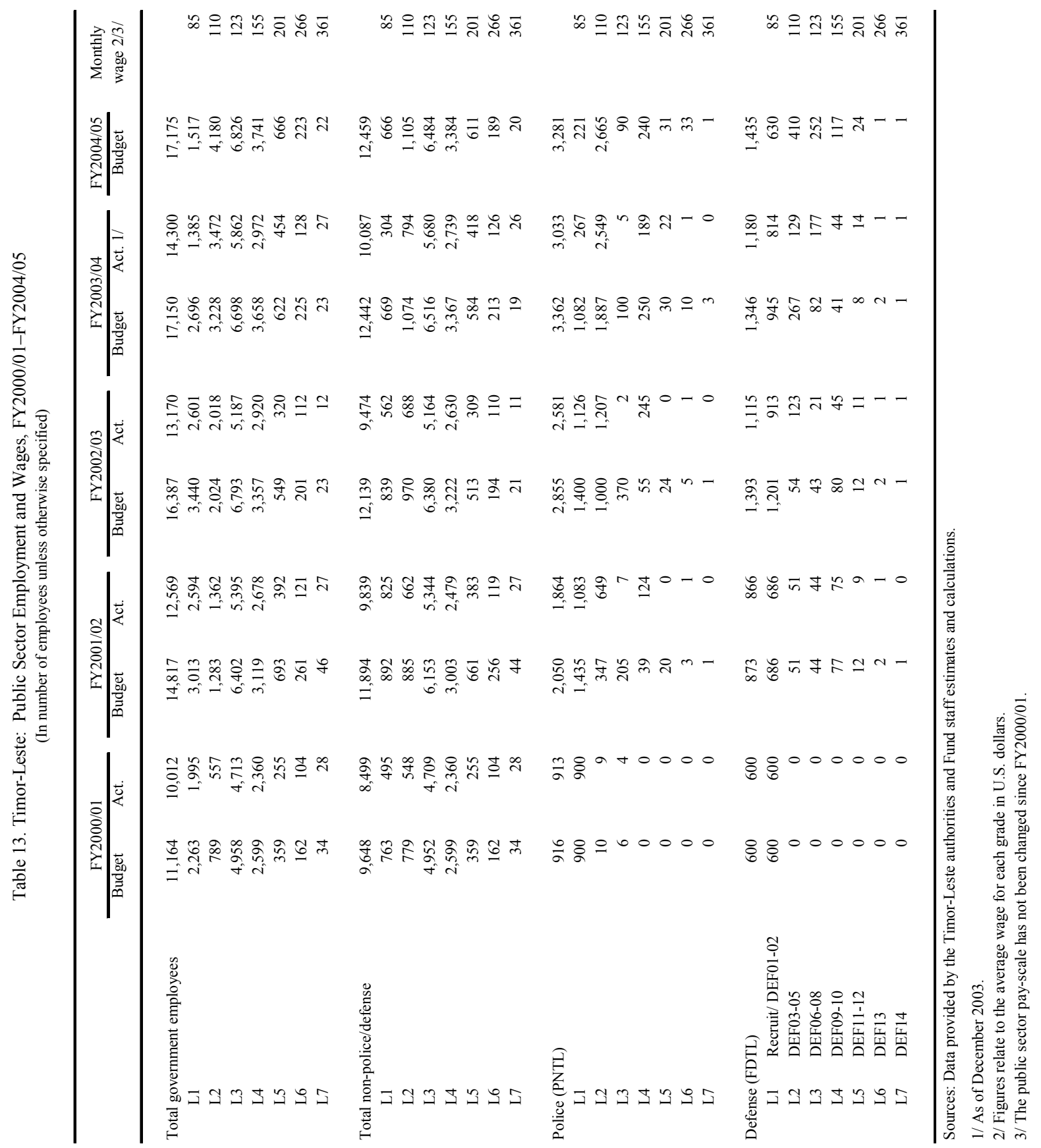


Table 14. Timor-Leste: Autonomous Agencies Operations, FY2000/01-FY2004/05 1/

\begin{tabular}{|c|c|c|c|c|c|c|c|}
\hline & \multirow[t]{2}{*}{ FY2000/01 } & \multirow[t]{2}{*}{ FY2001/02 } & \multirow[t]{2}{*}{ FY2002/03 } & \multicolumn{3}{|c|}{ FY2003/04 } & \multirow{2}{*}{$\frac{\text { FY2004/05 }}{\text { Budget 4/ }}$} \\
\hline & & & & Budget & Revised 2/ & Est. 3/ & \\
\hline & \multicolumn{7}{|c|}{ (In millions of U.S. dollars) } \\
\hline Total retained revenues & 1.4 & 4.4 & 4.9 & 6.9 & 5.9 & 5.3 & 8.4 \\
\hline Aviation & 0.4 & 1.1 & 1.0 & 0.9 & 0.9 & 1.1 & 0.9 \\
\hline Port & 0.7 & 1.3 & 1.1 & 0.7 & 0.7 & 0.8 & 1.0 \\
\hline Power & 0.3 & 2.0 & 2.9 & 5.3 & 4.3 & 3.4 & 6.5 \\
\hline Total expenditures & 8.8 & 9.1 & 12.6 & 13.5 & 13.5 & 12.7 & 13.6 \\
\hline Aviation & 0.1 & 0.2 & 0.8 & 0.9 & 0.9 & 0.9 & 0.9 \\
\hline Port & 0.1 & 0.2 & 0.5 & 0.5 & 0.5 & 0.5 & 0.6 \\
\hline Power & 8.6 & 8.7 & 11.2 & 12.1 & 12.1 & 11.3 & 12.1 \\
\hline Wages and salaries & 0.4 & 0.4 & 0.5 & 0.6 & 0.6 & 0.5 & 0.7 \\
\hline Aviation & 0.0 & 0.0 & 0.1 & 0.1 & 0.1 & 0.1 & 0.2 \\
\hline Port & 0.0 & 0.0 & 0.1 & 0.1 & 0.1 & 0.1 & 0.1 \\
\hline Power & 0.4 & 0.3 & 0.3 & 0.4 & 0.4 & 0.3 & 0.4 \\
\hline Goods and services & 7.8 & 7.9 & 9.5 & 12.0 & 12.0 & 11.3 & 12.1 \\
\hline Aviation & 0.0 & 0.1 & 0.5 & 0.5 & 0.5 & 0.5 & 0.6 \\
\hline Port & 0.0 & 0.1 & 0.2 & 0.2 & 0.2 & 0.2 & 0.2 \\
\hline Power & 7.8 & 7.6 & 8.8 & 11.3 & 11.3 & 10.6 & 11.3 \\
\hline Capital & 0.6 & 0.9 & 2.5 & 0.9 & 0.9 & 0.9 & 0.8 \\
\hline Aviation & 0.1 & 0.0 & 0.2 & 0.2 & 0.2 & 0.2 & 0.2 \\
\hline Port & 0.0 & 0.0 & 0.3 & 0.2 & 0.2 & 0.2 & 0.3 \\
\hline Power & 0.5 & 0.8 & 2.0 & 0.5 & 0.5 & 0.5 & 0.4 \\
\hline Overall balance & -7.4 & -4.7 & -7.7 & -6.6 & -7.6 & -7.4 & -5.3 \\
\hline Aviation & 0.3 & 0.9 & 0.2 & 0.0 & 0.0 & 0.2 & 0.0 \\
\hline Port & 0.6 & 1.1 & 0.5 & 0.2 & 0.2 & 0.3 & 0.4 \\
\hline Power & -8.4 & -6.8 & -8.3 & -6.8 & -7.8 & -7.9 & -5.7 \\
\hline Financing & 7.4 & 4.7 & 7.7 & 6.6 & 7.6 & 7.4 & 5.3 \\
\hline Transfers from the budget & 8.4 & 6.8 & 8.4 & 6.8 & 6.8 & 6.8 & 5.7 \\
\hline Aviation & 0.0 & 0.0 & 0.0 & 0.0 & 0.0 & 0.0 & 0.0 \\
\hline Port & 0.0 & 0.0 & 0.0 & 0.0 & 0.0 & 0.0 & 0.0 \\
\hline Power & 8.4 & 6.8 & 8.4 & 6.8 & 6.8 & 6.8 & 5.7 \\
\hline Other (Increase -) & -0.9 & -2.0 & -0.8 & -0.2 & 0.8 & 0.6 & -0.4 \\
\hline Aviation & -0.3 & -0.9 & -0.2 & 0.0 & 0.0 & -0.2 & 0.0 \\
\hline Port & -0.6 & -1.1 & -0.5 & -0.2 & -0.2 & -0.3 & -0.4 \\
\hline \multirow[t]{2}{*}{ Power } & 0.0 & 0.0 & -0.1 & 0.0 & 1.0 & 1.1 & 0.0 \\
\hline & \multicolumn{7}{|c|}{ (In percent of GDP) } \\
\hline Total retained revenues & 0.4 & 1.1 & 1.4 & 2.1 & 1.8 & 1.6 & 2.5 \\
\hline Aviation & 0.1 & 0.3 & 0.3 & 0.3 & 0.3 & 0.3 & 0.3 \\
\hline Port & 0.2 & 0.3 & 0.3 & 0.2 & 0.2 & 0.2 & 0.3 \\
\hline Power & 0.1 & 0.5 & 0.8 & 1.6 & 1.3 & 1.0 & 2.0 \\
\hline Total expenditures & 2.5 & 2.4 & 3.5 & 4.1 & 4.0 & 3.8 & 4.1 \\
\hline Aviation & 0.0 & 0.1 & 0.2 & 0.3 & 0.3 & 0.3 & 0.3 \\
\hline Port & 0.0 & 0.0 & 0.1 & 0.2 & 0.2 & 0.2 & 0.2 \\
\hline Power & 2.4 & 2.3 & 3.1 & 3.7 & 3.6 & 3.4 & 3.7 \\
\hline
\end{tabular}

Sources: Data provided by the Timor-Leste authorities, and Fund staff estimates and calculations.

1/ Fiscal year: July-June.

2/ Revised estimates made at the mid-year budget review (November 2003)

3/ Staff estimates based on the actual outcome through March 2004.

4/ Partially staff estimates. 
Table 15. Timor-Leste: Operations of the Power Authority, FY2000/01-FY2004/05 1/

\begin{tabular}{|c|c|c|c|c|c|c|c|}
\hline & \multirow[t]{2}{*}{ FY2000/01 } & \multirow[t]{2}{*}{ FY2001/02 } & \multirow[t]{2}{*}{ FY2002/03 } & \multicolumn{3}{|c|}{ FY2003/04 } & \multirow{2}{*}{$\frac{\text { FY2004/05 }}{\text { Budget }}$} \\
\hline & & & & Budget & Revised 2/ & Est. 3/ & \\
\hline & \multicolumn{7}{|c|}{ (In thousands of U.S. dollars) } \\
\hline Revenue collection 4/ & 258 & 1,997 & 2,871 & 5,300 & 4,300 & 3,376 & 6,450 \\
\hline Expenditure & 8,639 & 8,749 & 11,204 & 12,134 & 12,134 & 11,320 & 12,102 \\
\hline Wages and salaries & 364 & 309 & 312 & 389 & 389 & 307 & 416 \\
\hline Goods and services & 7,782 & 7,618 & 8,843 & 11,287 & 11,287 & 10,555 & 11,295 \\
\hline Fuel & 7,523 & 5,596 & 7,950 & 8,300 & 8,300 & 8,300 & 8,150 \\
\hline Maintenance & $\ldots$ & $\ldots$ & 386 & 1,500 & 1,500 & 1,433 & 1,800 \\
\hline Management contract fees & 0 & 0 & 0 & 1,200 & 1,200 & 535 & 1,000 \\
\hline Other & $\ldots$ & $\ldots$ & 507 & 287 & 287 & 287 & 345 \\
\hline Capital expenditure & 493 & 822 & 2,049 & 458 & 458 & 458 & 391 \\
\hline $\mathrm{O} / \mathrm{w}$ : Prepayment meter project 5/ & 0 & 0 & 1,900 & 0 & 0 & 0 & 0 \\
\hline Operating balance & $-7,887$ & $-5,929$ & $-6,284$ & $-6,376$ & $-7,376$ & $-7,486$ & $-5,261$ \\
\hline Overall balance & $-8,380$ & $-6,751$ & $-8,333$ & $-6,834$ & $-7,834$ & $-7,944$ & $-5,652$ \\
\hline Financing & 8,380 & 6,751 & 8,333 & 6,834 & 7,834 & 7,944 & 5,652 \\
\hline Budgetary transfers & 8,380 & 6,751 & 8,442 & 6,834 & 6,834 & 6,834 & 5,652 \\
\hline External assistance & 0 & 0 & 0 & 0 & 1,110 & 1,110 & 0 \\
\hline \multirow[t]{2}{*}{ Other } & 0 & 0 & -109 & 0 & -110 & 0 & 0 \\
\hline & \multicolumn{7}{|c|}{ (In percent of GDP) } \\
\hline Revenue collection $2 / 3 /$ & 0.1 & 0.5 & 0.8 & 1.6 & 1.3 & 1.0 & 2.0 \\
\hline Expenditure & 2.4 & 2.3 & 3.1 & 3.7 & 3.6 & 3.4 & 3.7 \\
\hline Wages and salaries & 0.1 & 0.1 & 0.1 & 0.1 & 0.1 & 0.1 & 0.1 \\
\hline Goods and services & 2.2 & 2.0 & 2.4 & 3.4 & 3.4 & 3.2 & 3.4 \\
\hline Capital expenditure & 0.1 & 0.2 & 0.6 & 0.1 & 0.1 & 0.1 & 0.1 \\
\hline \multirow[t]{2}{*}{ Budgetary transfers } & 2.4 & 1.8 & 2.3 & 2.1 & 2.0 & 2.0 & 1.7 \\
\hline & \multicolumn{7}{|c|}{ (In percent of total CFET expenditure) } \\
\hline \multirow[t]{2}{*}{ Budgetary transfers } & 16.3 & 12.8 & 11.9 & 8.6 & 9.2 & 9.2 & 7.5 \\
\hline & \multicolumn{7}{|c|}{ (In thousands of U.S. dollars) } \\
\hline \multicolumn{8}{|l|}{ Memorandum item: } \\
\hline Average monthly revenue collection & 29 & 166 & 239 & 442 & 358 & 281 & 538 \\
\hline
\end{tabular}

Sources: Data provided by the Timor-Leste authorities, and Fund staff estimates and calculations.

1/ Fiscal year: July-June.

2/ Revised estimates made at the mid-year budget review (November 2003).

3/ Staff estimates based on the actual outcome through March 2004.

4/ Includes interest income; electricity bill collection started in October 2000.

$5 /$ The total cost of prepayment meter project was $\$ 2.7$ million, of which $\$ 1.9$ million was provided from the budget, with the remainder financed by Norad and TFET. 
Table 16. Timor-Leste: Combined Sources Fiscal Operations, FY2000/01-FY2003/04 1/

\begin{tabular}{|c|c|c|c|c|}
\hline & FY2000/01 & FY2001/02 & FY2002/03 & $2003 / 04$ \\
\hline & \multicolumn{4}{|c|}{ Est. } \\
\hline & \multicolumn{4}{|c|}{ (In millions of U.S. dollars) } \\
\hline Revenue & 29 & 36 & 54 & 65 \\
\hline CFET & 27 & 31 & 49 & 59 \\
\hline Autonomous agencies & 1 & 4 & 5 & 6 \\
\hline Total expenditure & 432 & 463 & 378 & 305 \\
\hline CFET 2/ & 43 & 46 & 62 & 67 \\
\hline Non-CFET & 389 & 417 & 317 & 238 \\
\hline Autonomous agencies & 9 & 9 & 11 & 15 \\
\hline TFET & 47 & 46 & 25 & 20 \\
\hline Bilateral & 139 & 161 & 153 & 126 \\
\hline UNTAET/UNMISET 3/ & 194 & 201 & 128 & 77 \\
\hline Recurrent expenditure 4/ & 340 & 374 & 297 & 241 \\
\hline CFET 2/ & 22 & 35 & 47 & 56 \\
\hline Non-CFET & 318 & 339 & 250 & 184 \\
\hline Autonomous agencies & 8 & 8 & 9 & 13 \\
\hline TFET & 24 & 23 & 12 & 10 \\
\hline Bilateral & 92 & 106 & 101 & 84 \\
\hline UNTAET/UNMISET 3/ & 194 & 201 & 128 & 77 \\
\hline Capital expenditure & 92 & 89 & 81 & 65 \\
\hline CFET $2 /$ & 21 & 11 & 15 & 11 \\
\hline Non-CFET & 71 & 78 & 66 & 54 \\
\hline Autonomous agencies & 1 & 1 & 2 & 1 \\
\hline TFET & 23 & 23 & 12 & 10 \\
\hline Bilateral & 47 & 55 & 52 & 43 \\
\hline UNTAET/UNMISET 3/ & 0 & 0 & 0 & 0 \\
\hline Overall balance & -403 & -428 & -324 & -241 \\
\hline Financing & 403 & 428 & 324 & 241 \\
\hline CFET 2/ & 23 & 19 & 19 & 17 \\
\hline Non-CFET & 380 & 408 & 305 & 224 \\
\hline TFET & 47 & 46 & 25 & 20 \\
\hline Bilateral & 139 & 161 & 153 & 126 \\
\hline \multirow[t]{2}{*}{ UNTAET/UNMISET 3/ } & 194 & 201 & 128 & 77 \\
\hline & \multicolumn{4}{|c|}{ (In percent of GDP) } \\
\hline Revenue & 8 & 9 & 15 & 19 \\
\hline Total expenditure & 122 & 121 & 105 & 91 \\
\hline CFET 2/ & 12 & 12 & 17 & 20 \\
\hline Non-CFET & 110 & 109 & 88 & 71 \\
\hline Recurrent expenditure 4/ & 96 & 97 & 82 & 72 \\
\hline CFET 2/ & 6 & 9 & 13 & 17 \\
\hline Non-CFET & 90 & 88 & 69 & 55 \\
\hline Capital expenditure & 26 & 23 & 22 & 19 \\
\hline CFET & 6 & 3 & 4 & 3 \\
\hline Non-CFET & 20 & 20 & 18 & 16 \\
\hline Overall balance & -114 & -111 & -90 & -72 \\
\hline Financing & 114 & 111 & 90 & 72 \\
\hline CFET & 7 & 5 & 5 & 5 \\
\hline Non-CFET & 107 & 106 & 85 & 67 \\
\hline
\end{tabular}

Sources: Data provided by the Timor-Leste authorities; and Fund staff estimates.

1/ Include expenditure programs undertaken by bilateral donors, UNTAET/UNMISET, and international financial institutions outside the central government budget. The coverarge of UNTAET/UNMISET operations differ from the official budget presentation.

2/ Net of CFET budget transfers to autonomous agencies.

3/ Excludes military expenditures and those not directly related to government operations.

4/ Includes technical assistance. 
Table 17. Timor-Leste: Balance of Payments, 1999-2003

\begin{tabular}{|c|c|c|c|c|c|}
\hline & 1999 & 2000 & 2001 & 2002 & 2003 \\
\hline & \multicolumn{5}{|c|}{ Est. } \\
\hline & \multicolumn{5}{|c|}{ (In millions of U.S. dollars) } \\
\hline Current account excl. official transfers & -92 & -279 & -309 & -292 & -230 \\
\hline Current account incl. official transfers & 6 & 48 & 54 & 44 & 43 \\
\hline Trade balance & -67 & -235 & -264 & -245 & -195 \\
\hline Exports of goods 1/2/ & 52 & 5 & 4 & 6 & 7 \\
\hline O/w: Coffee & $\ldots$ & 4 & 3 & 5 & 6 \\
\hline Imports of goods $1 /$ & -119 & -240 & -268 & -251 & -203 \\
\hline $\mathrm{O} / \mathrm{w}$ : International assistance-related & -58 & -125 & -129 & -126 & -104 \\
\hline Services (net) & -25 & -48 & -49 & -48 & -40 \\
\hline Income (net) & 0 & 3 & 5 & 1 & 6 \\
\hline $\mathrm{O} / \mathrm{w}: \mathrm{Oil} /$ gas royalty and interest & 0 & 3 & 4 & 0 & 4 \\
\hline Current transfers (net) & 98 & 327 & 363 & 336 & 273 \\
\hline O/w: Oil/gas tax revenues & 0 & 5 & 8 & 16 & 27 \\
\hline International assistance & 43 & 315 & 347 & 314 & 240 \\
\hline Capital and financial accounts & -3 & -54 & -61 & -36 & -16 \\
\hline Official capital transfers & 0 & 71 & 74 & 71 & 58 \\
\hline Financial accounts & -3 & -125 & -134 & -107 & -74 \\
\hline Errors and omissions (net) & -3 & 23 & 14 & 12 & -10 \\
\hline Overall balance & 0 & 16 & 8 & 20 & 18 \\
\hline Changes in foreign assets (increase -) & 0 & -16 & -8 & -20 & -18 \\
\hline Oil/gas revenue savings (increase -) & 0 & -3 & -4 & 0 & -3 \\
\hline Other & 0 & -13 & -3 & -20 & -15 \\
\hline & \multicolumn{5}{|c|}{ (In percent of GDP) } \\
\hline Current account excl. official transfers & -34 & -87 & -80 & -77 & -67 \\
\hline Current account incl. official transfers & 2 & 15 & 14 & 12 & 13 \\
\hline Trade balance & -25 & -73 & -68 & -64 & -57 \\
\hline & \multicolumn{5}{|c|}{ (In millions of U.S. dollars) } \\
\hline \multicolumn{6}{|l|}{ Memorandum items: } \\
\hline Oil/gas revenues & 0 & 8 & 13 & 17 & 31 \\
\hline Gross foreign assets (end-period) & 0 & 16 & 24 & 44 & 61 \\
\hline O/w: Oil/gas revenue savings & 0 & 3 & 7 & 7 & 11 \\
\hline
\end{tabular}

Source: Data provided by the Timor-Leste authorities, and Fund staff estimates.

1/ Figures before 2000 include unrecorded border trade.

2/ Exclude oil/gas revenues, which are recorded under the income (royalties) and transfers (tax revenues) because of lack of detailed data on the oil/gas sector. 
Table 18. Timor-Leste: Oil/Gas Receipts, 2000-2003

\begin{tabular}{|c|c|c|c|c|}
\hline & 2000 & 2001 & 2002 & 2003 \\
\hline & \multicolumn{4}{|c|}{ Est. } \\
\hline & \multicolumn{4}{|c|}{ (In millions of U.S. dollars) } \\
\hline Total oil/gas receipts & 8.1 & 12.5 & 16.5 & 31.2 \\
\hline Royalties (first tranche petroleum) $1 /$ & 3.1 & 4.2 & 0.0 & 4.3 \\
\hline Elang-Kakatua-Kakatua-North field & 3.1 & 4.2 & 0.0 & 4.3 \\
\hline Bayu-Undan field & 0.0 & 0.0 & 0.0 & 0.0 \\
\hline Tax revenues & 5.0 & 8.2 & 16.4 & 26.8 \\
\hline Elang-Kakatua-Kakatua-North field & 5.0 & 8.2 & 7.4 & 9.7 \\
\hline Bayu-Undan field & 0.0 & 0.0 & 9.0 & 17.0 \\
\hline \multirow[t]{2}{*}{ Interest income } & 0.0 & 0.1 & 0.1 & 0.1 \\
\hline & \multicolumn{4}{|c|}{ (In percent of GDP) } \\
\hline Total oil/gas receipts & 2.5 & 3.2 & 4.3 & 9.1 \\
\hline Royalties (first tranche petroleum) $1 /$ & 1.0 & 1.1 & 0.0 & 1.3 \\
\hline Elang-Kakatua-Kakatua-North field & 1.0 & 1.1 & 0.0 & 1.3 \\
\hline Bayu-Undan field & 0.0 & 0.0 & 0.0 & 0.0 \\
\hline Tax revenues & 1.5 & 2.1 & 4.3 & 7.9 \\
\hline Elang-Kakatua-Kakatua-North field & 1.5 & 2.1 & 2.0 & 2.9 \\
\hline Bayu-Undan field & 0.0 & 0.0 & 2.4 & 5.0 \\
\hline
\end{tabular}

Sources: Data provided by the Timor-Leste authorities; and Fund staff estimates.

1/ May not correspond to flow data calculated from monetary statistics (Timor Sea Account, Table 7) due to differences in the timing of data recording. 


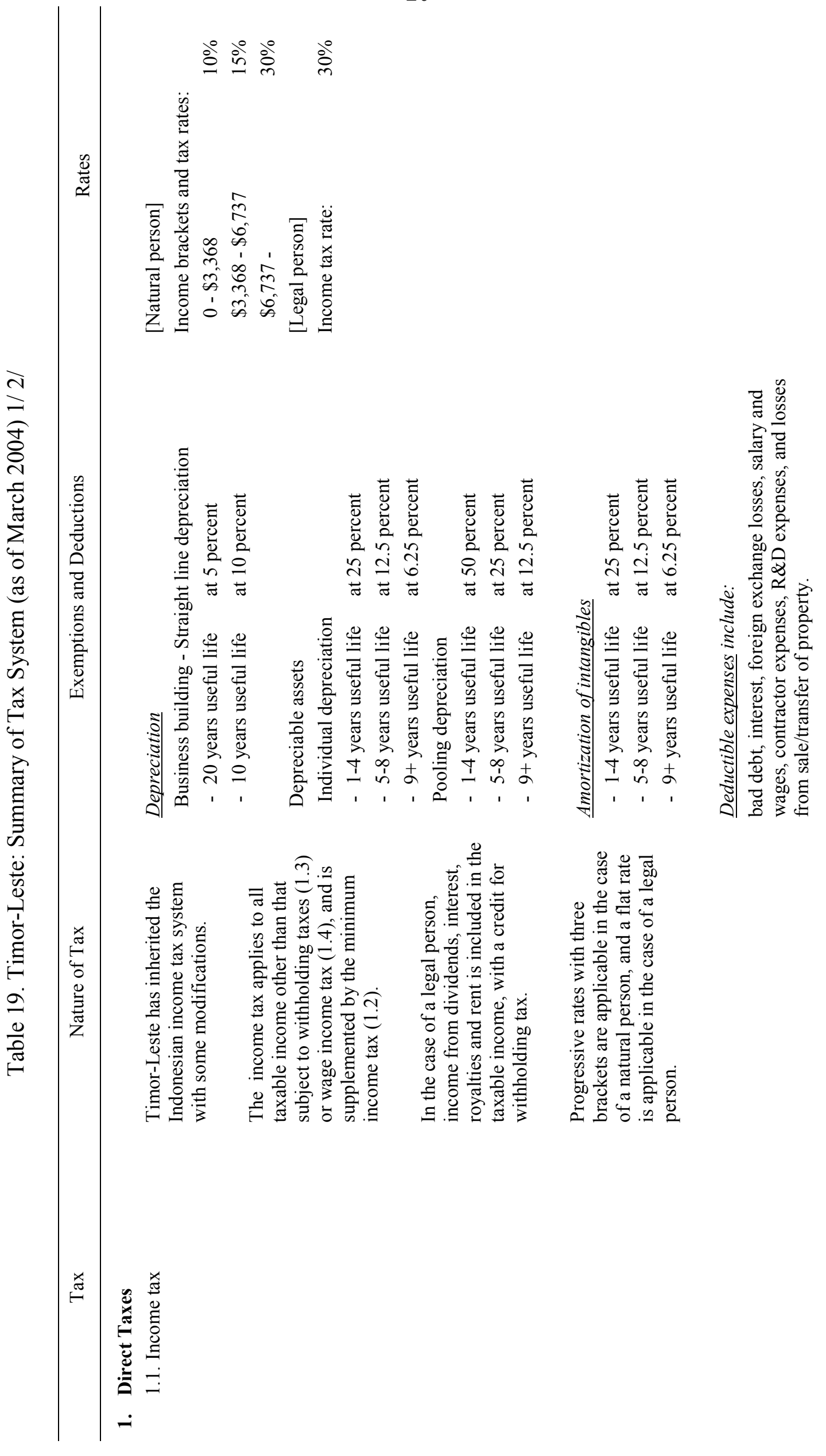


-21 -

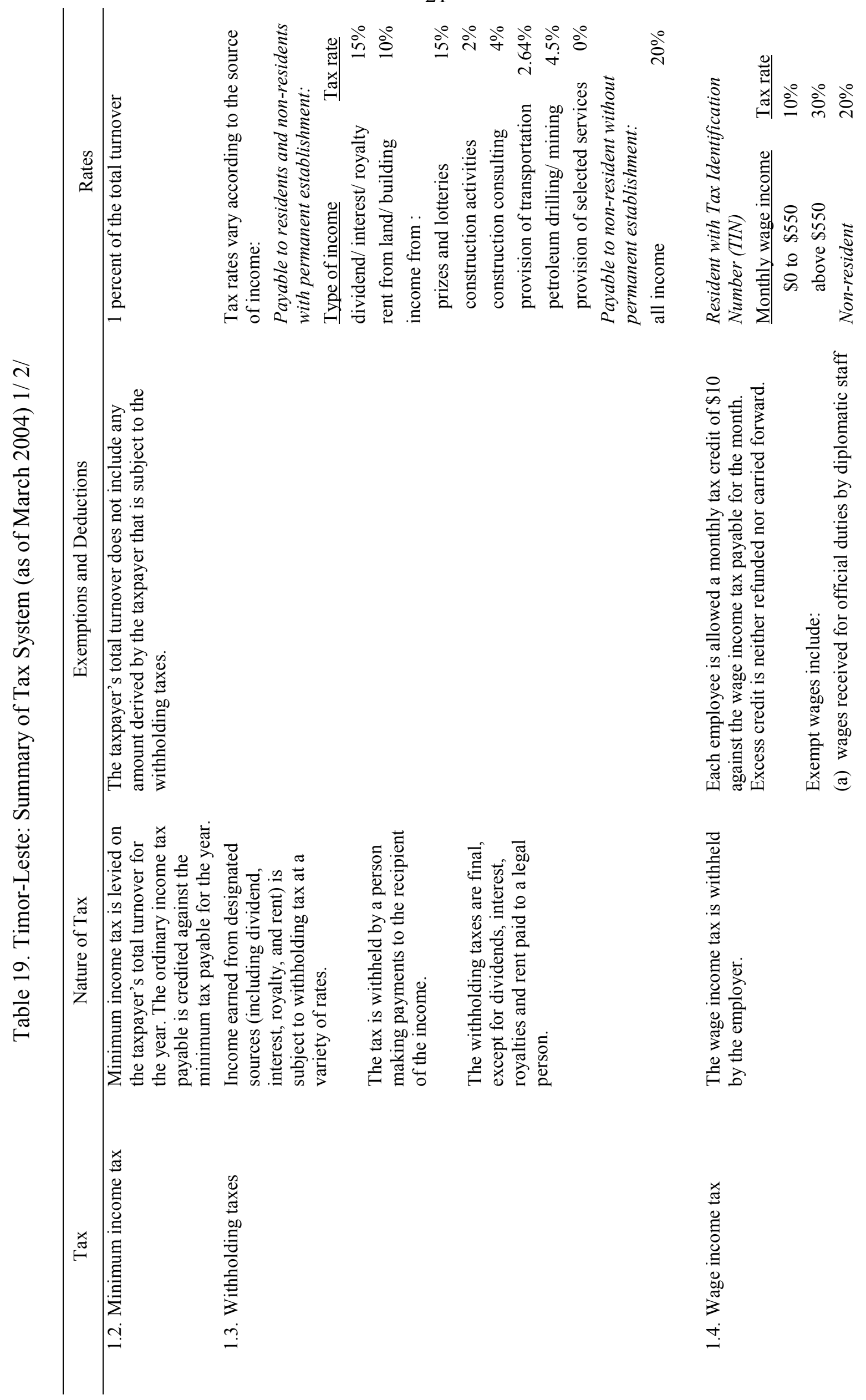




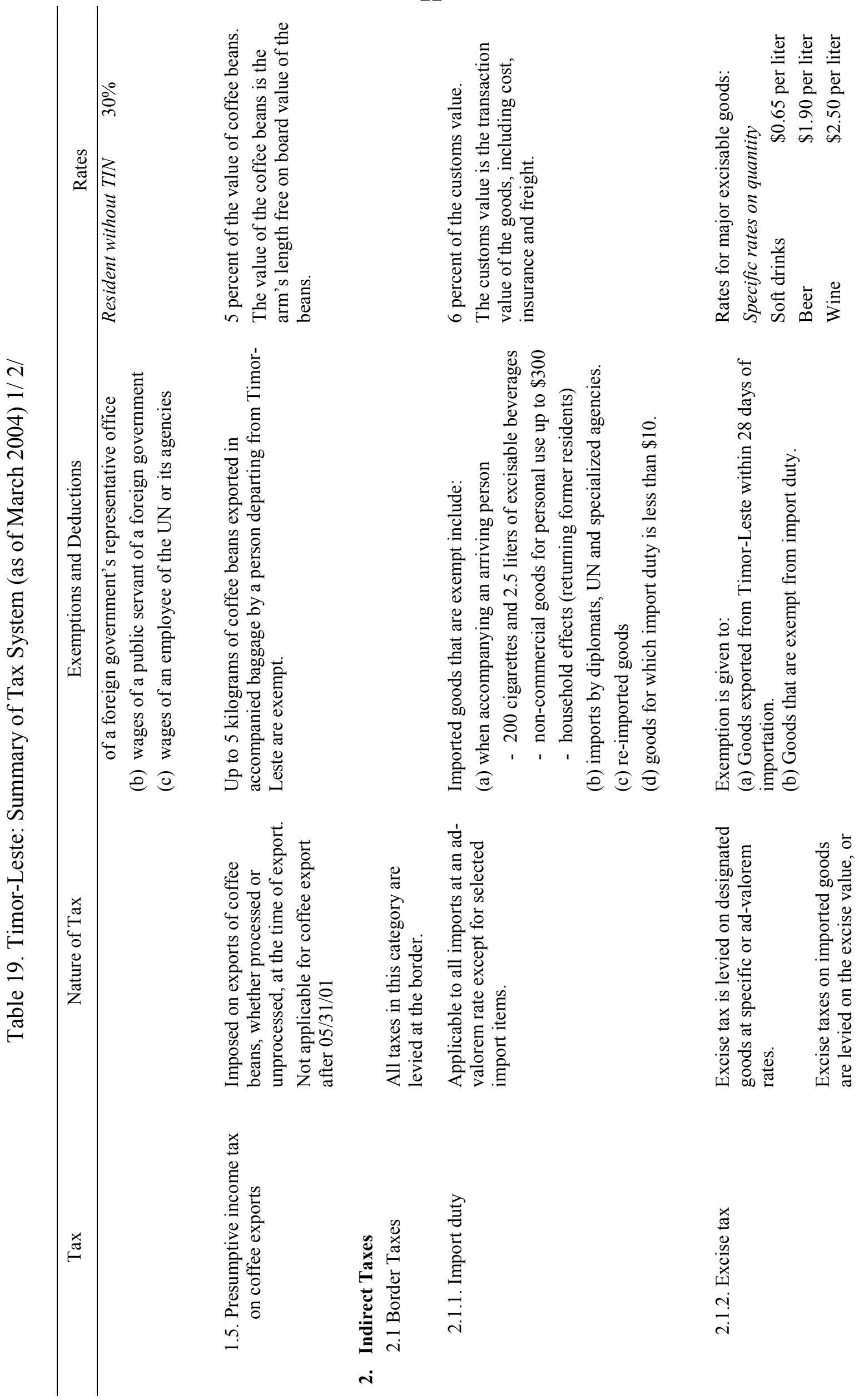




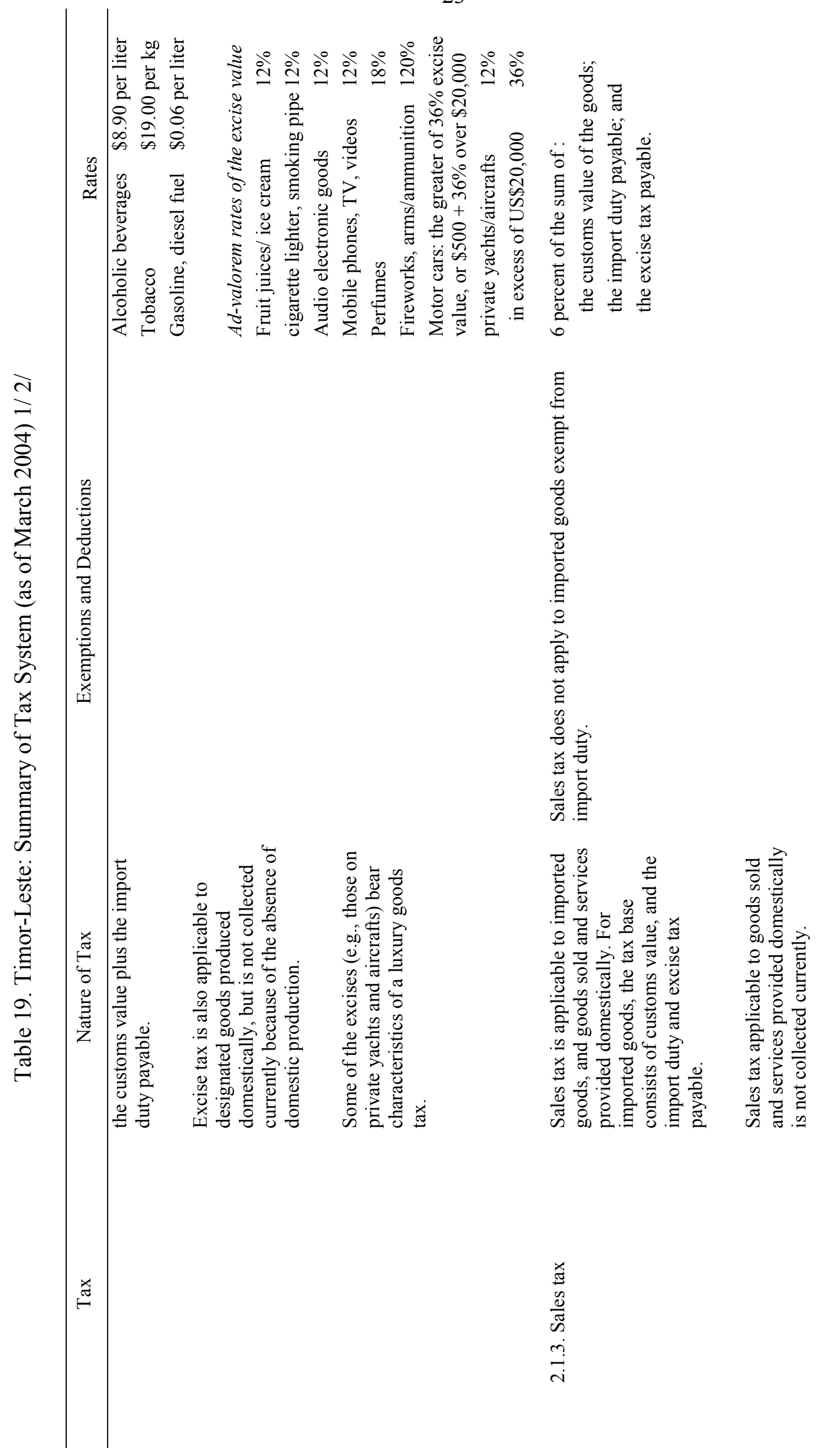




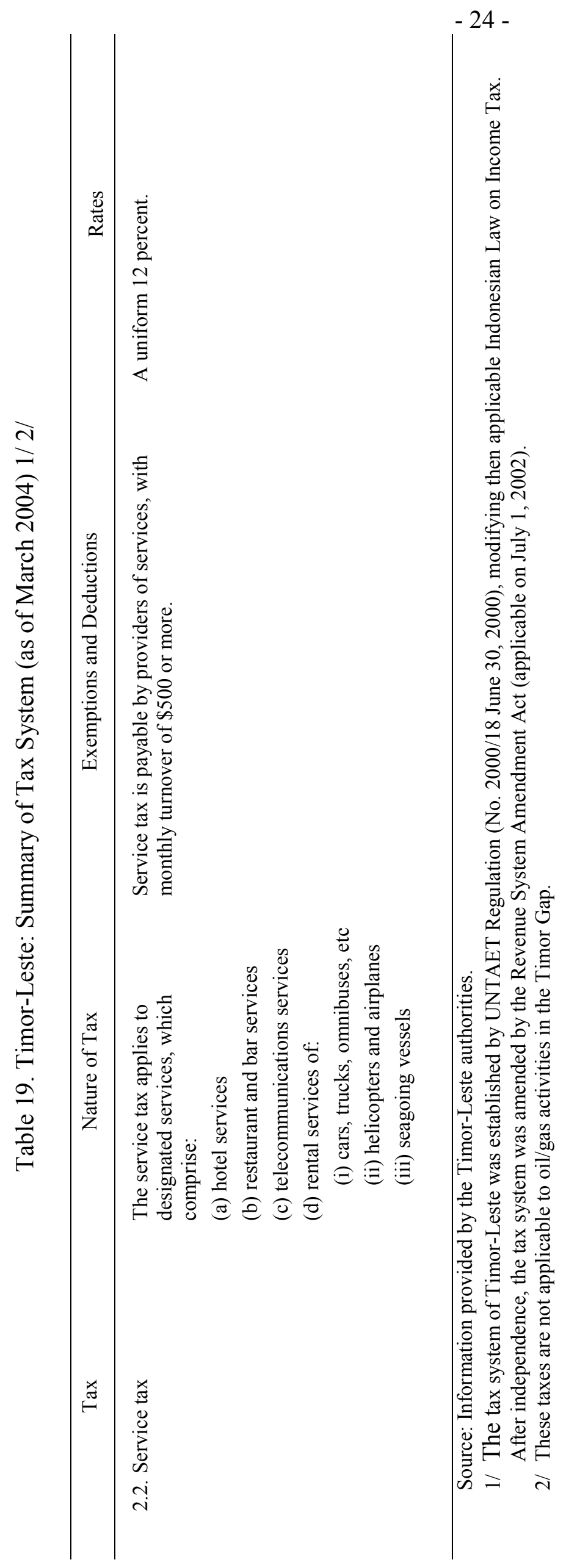


Table 20. Timor-Leste: Summary of the Exchange and Payments System

(Position as of April 30, 2004)

\section{Status Under IMF Articles of Agreement}

1. Date of membership Article VIII

\section{Currency}

a. Other legal tender

2. Exchange rate structure

a. Unitary

3. Classification

a. Exchange arrangement with no separate legal tender

\section{Exchange tax}

5. Exchange subsidy

6. Forward exchange market

a. Official cover of forward operations

\section{Prescription of currency} requirements

\author{
a. Controls on the use of \\ domestic currency \\ b. Use of foreign exchange \\ among residents
}

\section{Payments arrangements}

3. Administration of control

\section{International security} restrictions

\section{Payments arrears}

6. Controls on trade in gold (coins and/or bullion)

7. Controls on exports and
July 23, 2002.

Date of acceptance: July 23, 2002

\section{Exchange Arrangement}

The currency of the Democratic Republic of Timor Leste is the U.S. dollar.

Yes On November 10, 2003, Timorese coins were introduced to serve as fractional currency to the U.S. dollar.

Yes

Yes

The dollar is legal tender and circulates freely. Foreign exchange transactions are effected through three (foreign-owned) commercial banks and one licenced currency exchange bureau.

No

No

No

\section{Arrangements for Payments and Receipts}

All domestic transactions and settlements must be in the domestic currency (U.S. dollar).

Withdrawals from bank accounts denominated in foreign currencies must be made in U.S. dollars, though bank transfers from foreign currency accounts abroad may be made in foreign currencies.

None

Overall responsibility for the administration of exchange controls rests with the BPA, which has the power to regulate payment and settlement systems in domestic and foreign currency.

No

No

No 
imports of banknotes

a. On exports

b. On imports

1. Domestic currency

2. Foreign currency

No

No

Approval required

b. Held abroad

Approval required

2. Accounts in domestic currency held abroad

3. Accounts in domestic currency convertible into foreign currency

1. Foreign exchange accounts permitted

a. Approval required

2. Domestic currency accounts

a. Convertible into foreign currency

b. Approval required

3. Blocked accounts

No

No

\section{Imports and Import Payments}

1. Foreign exchange budget

No

2. Financing requirements for imports

3. Documentation requirements for release of foreign exchange for imports

4. Import licenses and other No nontariff measures

restrictions

\section{Nonresident Accounts}

No

restrictions

No

No

restrictions

No

No

No
No restriction

\section{Resident Accounts}

There are no restrictions on the holding of foreign exchange accounts, but withdrawals from the accounts should be made in domestic currency (U.S. dollar).

No

No

No

No restricitons

Restricted

Withdrawals from bank accounts in foreign currency are prohibited.

Withdrawals from bank accounts in foreign currency are prohibited. 
5. Import taxes and/or tariffs

a. Taxes collected through the exchange system

6. State import monopoly

No

\section{Exports and Export Proceeds}

1. Repatriation requirements

2. Financing requirements

3. Documentation requirements

4. Export licenses

5. Export taxes
No

No

No

No

No
There are no quantitative restrictions on imports. With the exception of selected items (e.g., cigarettes and alcohol with certain limits, household effects of returning former residents), a uniform ad-valorem tariff ( 6 percent) is levied on all imports. Also, excise taxes are levied on imports of selected goods at specific or ad-valorem rates (10170 percent) depending on types of goods. In addition, the sale tax (6 percent) is levied on the sum of customs value, import duty, and excise payable.

\section{Payments for Invisible Transactions and Current Transfers}

Controls on transfers

No

\section{Proceeds from Invisible Transactions and Current Transfers}

1. Repatriation requirements

2. Restrictions on use of funds
No

No

\section{Capital Transactions}

\section{A. Controls on capital} transactions

1. Controls on capital and money market instruments

No

No domestic capital and money markets have developed yet.
2. Controls on derivatives No and other instruments

3. Controls on credit operations

4. Controls on direct investment

5. Controls on liquidation of No direct investment

6. Controls on real estate transactions
No

No 
a. Purchase abroad by
residents
b. Purchase locally by
nonresidents
c. Sale locally by
nonresidents
7. Controls on personal
capital transactions
8. Provisions specific to
commercial banks and
other credit institutions

a. Borrowing abroad

b. Maintenance of accounts abroad

c. Lending to nonresidents (financial or commercial credits)

d. Lending locally in foreign Yes exchange

e. Purchase of locally issued Yes securities denominated in foreign exchange

f. Differential treatment of deposit accounts in foreign exchange

g. Differential treatment of deposit accounts held by nonresidents

h. Investment regulations

i. Open foreign exchange position limits

9. Provisions specific to institutional investors

10. Other controls imposed by securities laws

Exchange arrangement

Arrangements for payments and receipts
Nov. 10

No

No

No

No

No

No

No

No

No

Nov. 13
No

Yes

Yes

The constitution prohibits ownership of land by foreigners.

The constitution prohibits ownership of land by foreigners.
All domestic transactions must be made in the domestic currency.

All domestic transactions must be denominated in the domestic currency.

\section{Changes During 2003}

Timorese coins were introduced to serve as fractional currency to the U.S. dollar.

A new currency law revoked the restrictions on importing foreign currencies. (Previously, imports of foreign currencies exceeding the equivalent of $\$ 2,000$ are prohibited without a permit from the BPA. Also imports of currencies issued by countries which maintain export restrictions were limited to the equivalent of $\$ 500$ without BPA approval.) 